\title{
Addendum
}

\section{Addendum to Evidence for the Detection of Non-Endotoxin Pyrogens by the Whole Blood Monocyte Activation Test}

Nina Hasiwa ${ }^{1,2}$, Mardas Daneshian ${ }^{1}$, Peter Bruegger ${ }^{4}$, Stefan Fennrich ${ }^{5}$, Astrid Hochadel ${ }^{2}$, Sebastian Hoffmann ${ }^{6}$, Felix E. Rivera-Mariani ${ }^{3}$, Christoph Rockel ${ }^{7}$, Stefanie Schindler ${ }^{8}$, Ingo Spreitzer ${ }^{9}$, Sandra Stoppelkamp ${ }^{5}$, Kranthi Vysyaraju ${ }^{3}$, and Thomas Hartung ${ }^{1,3}$

${ }^{1}$ CAAT-Europe, University of Konstanz, Konstanz, Germany; ${ }^{2}$ AtaX-Advice, Konstanz, Germany; ${ }^{3}$ Johns Hopkins University, Bloomberg School of Public Health, CAAT, Baltimore, USA; ${ }^{4}$ Novartis, Basel, Switzerland; ${ }^{5}$ Department of Thoracic, Cardiac and Vascular Surgery, Tübingen University Hospital, Tübingen, Germany; ${ }^{6}$ seh consulting + services, Paderborn, Germany; ${ }^{7}$ Enzler Hygiene AG, Center for Hygiene, Pratteln, Switzerland; ${ }^{8}$ Animalfree Research, Zürich, Switzerland; ${ }^{9}$ Paul-Ehrlich Institut (PEI), Langen, Germany

Regarding this $\mathrm{t}^{4}$ report, which appeared in ALTEX (2013), 30(2), Thomas Hartung would like to acknowledge that he received a honorarium from Merck-Millipore for his efforts in steering the article, which was offered to him only after the article was published and thus originally not disclosed. 